\title{
Representación semántica del término 'psicología médica' en estudiantes de medicina: un estudio longitudinal
}

\author{
Ileana Petra-Micu, Jacqueline Cortés-Morelos, José Antonio Talayero-Uriarte, Mariana Fouilloux-Morales
}

Introducción. La psicología médica constituye una parte esencial en la formación integral del alumno de medicina. Poco se ha estudiado sobre la manera en que éste la concibe.

objetivo. Analizar el proceso de estructuración y reestructuración que el estudiante de medicina realiza en torno al concepto de 'psicología médica'.

Sujetos y métodos. La muestra de estudio estuvo integrada por un grupo de alumnos de la licenciatura de médico cirujano de la Facultad de Medicina de la Universidad Nacional Autónoma de México, perteneciente al Programa de Alta Exigencia Académica, y al que se dio seguimiento durante los cinco años de sus estudios. Se llevó a cabo un estudio observacional, longitudinal y prospectivo con cortes transversales anuales, con un análisis cuantitativo y cualitativo de la información obtenida, utilizando el método de redes semánticas naturales.

Resultados. A partir de las ocho palabras que los alumnos asociaron con el término estudiado y que prevalecieron a lo largo de los cinco años de la investigación, se obtuvo la siguiente definición: 'La psicología médica considera a la mente del paciente dentro de la relación de ayuda por parte del médico, que comprende su personalidad en pro de la salud'.

Conclusiones. El estudio sistemático de la asignatura 'Psicología Médica' parece brindar al alumno recursos que favorecen la consolidación de términos que permanecen a lo largo de sus estudios y que se reflejarán en una futura práctica profesional más humanista.

Palabras clave. Estudiante. Medicina. Psicología médica. Redes semánticas naturales.

\section{Semantic representation of the term 'medical psychology' in medical students: a longitudinal study}

Introduction. Medical psychology constitutes an important part of the formation of a medical student and little has been studied on the way these students conceive it.

Aim. To analyze the structural and restructuring process in medical students of the term 'medical psychology'.

Subjects and methods. The sample was formed by a group of students from the Faculty of Medicine of the National Autonomous University of Mexico, who belonged to a Higher Academic Demands Course and was followed during their five years of studies. It was a prospective, observational, and longitudinal with annual cross-sections study, with quantitative and qualitative analysis of the information obtained through the implementation of the natural semantic network method.

Results. From the eight words that were constant throughout the years, the next definition emerged: 'medical psychology considers that the mind of the patient in relation to the help the doctor gives helps to understand his personality in favor of his health'.

Conclusions. The systematic study the subject of 'Medical Psychology' seems to give the students resources to help consolidate terms that will be with him all along his studies and hopefully will permit him to develop a humanistic attitude that will remain with him during his professional life.

Key words. Medical psychology/behavioral sciences. Medicine. Natural semantic network. Student.

\section{Introducción}

La psicología médica se inicia como asignatura en Europa a mediados del siglo xIx. En uno de los primeros libros de psicología médica, en 1922, Kretsch- mer [1] destacó la necesidad de una preparación psicológica de los alumnos de medicina a través de la enseñanza de una psicología médica inspirada tanto en la psicología normal como en la patológica, reservando el mayor espacio al plano afectivo
Departamento de Psiquiatría y Salud Mental. Facultad de Medicina. Universidad Nacional Autónoma de México. México DF, México.

Correspondencia: Dra. lleana Petra Micu. Departamento de Psiquiatría y Salud Mental. Facultad de Medicina. Universidad Nacional Autónoma de México. Circuito Escolar, s/n. Facultad de Medicina, edif. F. Ciudad Universitaria. CP 04510. México DF, México.

E-mail:

ileanapetra@yahoo.com

Conflicto de intereses: No declarado.

Competing interests: None declared.

(c) 2015 FEM 
dinámico. Todo esto, agregaría Schneider [2], con la finalidad de comprender mejor al enfermo.

En México, De la Fuente [3] define la psicología médica como un campo de la psicología aplicada que reúne conocimientos y provee conceptos explicativos y criterios clínicos sobre los aspectos psicológicos de los problemas médicos y de la labor del médico; se trata de una disciplina clínica que se aproxima al hombre sano y enfermo como una totalidad; se alimenta de sus propias observaciones y recibe aportaciones de las ciencias biomédicas, así como de la ciencias humanas (sociología, antropología, axiología, etcétera).

En este mismo sentido, otros autores coinciden en que la psicología médica brinda los conocimientos de la psicología que son útiles para el ejercicio de la medicina integral, en especial los conocimientos, habilidades y actitudes generales, vinculados con su dominio de la relación médico-paciente y su comprensión amplia de la enfermedad como hecho humano $[4,5]$.

La relevancia de la psicología médica en la enseñanza de la medicina se refleja a través de sus contenidos curriculares. Así, en la Facultad de Medicina de la Universidad Nacional Autónoma de México (UNAM), el plan de estudios incluye la enseñanza de la psicología médica en el primer y tercer año de la licenciatura, abarcando temas como: 'Introducción al estudio de la medicina biopsicosocial,' 'El estudio de la personalidad,' 'El papel de la familia en el desarrollo del individuo,' 'El ciclo vital,' 'La relación médico-paciente', 'El examen médico-psicológico' y 'Comunicación', entre otros.

Ante estos temas señalados, cabría preguntarse si los contenidos curriculares influyen en la manera en que los estudiantes de medicina perciben la psicología médica durante su carrera. Es posible analizar cómo la conceptualizan, a través de diferentes opciones. A partir de la psicología cognitiva, se han generado una serie de modelos que posibilitan el análisis cualitativo de la semántica [6-9], entre los cuales destaca el de las redes semánticas, que se ha utilizado en distintos contextos $[10,11]$, incluido el de la medicina $[12,13]$.

El modelo de las denominadas 'redes semánticas naturales' (RSN) resulta de gran utilidad en el análisis de las palabras que definen un concepto en términos del valor, fuerza, peso o densidad que tienen en la construcción de redes semánticas en torno al concepto aprendido [14]. Este modelo ofrece la posibilidad de conocer y fortalecer (o modificar) las estructuras y procesos del pensamiento, mediante el análisis de sus resultados, siendo de fácil aplicación por poseer un carácter dinámico al interactuar los contenidos y la estructura de la red semántica con otros procesos de la memoria [15].

Las RSN se han empleado en diferentes ámbitos y poblaciones para observar la forma en que las personas perciben o conceptualizan diferentes aspectos de su entorno académico, político o social, con fines de índole exploratoria y descriptiva o para promover estrategias de acción con base en lo observado [16-18]. En México, por ejemplo, se han utilizado en población general y jóvenes, para identificar la forma en que construyen y reconstruyen el significado que asignan a distintos conceptos de su entorno [19-22]; en estudiantes universitarios, para evaluar su evolución en el aprendizaje de contenidos académicos [23], y en médicos y psicólogos, para conocer el significado que éstos atribuyen a la depresión [24].

La utilidad de estudiar las palabras definidoras por el método de RSN en el ámbito académico se puede resumir en:

- La valoración de los conocimientos previos que sobre la materia poseen los alumnos, así como de sus habilidades académicas, sus procesos de autorregulación, su capacidad para el procesamiento, su conocimiento selectivo y la experiencia que tienen sobre una tarea específica.

- El uso de la información para la toma de decisiones sobre la enseñanza, organización de los contenidos programados, selección de textos e instrumentación de estrategias de aprendizaje.

- La evaluación de la evolución de los conceptos, de su estructura y de su jerarquización a lo largo de la carrera.

Evaluar si el estudiante es capaz de formular una definición propia de lo que entiende por psicología médica limitaría con mucho lo que se puede investigar de los cambios en la estructuración del concepto que se va integrando a lo largo de la carrera, pues con frecuencia repetiría el concepto inicial. Por esta razón, solicitar al alumno que asocie el término 'psicología médica' con otras palabras resulta muy enriquecedor y permite comprender la manera en que se conforma el pensamiento, el deber ser y el hacer del futuro médico.

El presente estudio tiene como finalidad analizar el proceso de estructuración y reestructuración del concepto de 'psicología médica', mediante las palabras definidoras utilizadas por los estudiantes, identificando cómo está organizado el concepto cuando ingresan a la carrera y al concluir su quinto año de estudio, después de haber estado expuestos a la influencia de las ciencias médicas, sus ramas auxiliares y sus fundamentos filosóficos y éticos. 


\section{Sujetos y métodos}

El método empleado fue el de RSN, en el cual se solicita al alumno escribir las palabras que considera que tienen una mayor relación o cercanía al concepto clave en estudio; por ejemplo, si nos interesara saber cómo conceptúa el término 'libro', el encuestado podría responder con una lista de sustantivos, verbos o adjetivos tales como 'capítulo, 'índice', 'lectura, 'aprendizaje', 'ideas', etc. No existe un número límite de palabras a escribir, sólo se le pide que la lista no incluya frases ni oraciones. Las palabras así listadas deben jerarquizarse de forma progresiva, otorgando el valor de uno a la palabra que considera más importante o explicativa del término. En el ejemplo mencionado, el número uno podría ser la palabra 'lectura'; el dos, 'ideas', y así sucesivamente.

Dentro del análisis de las RSN se manejaron los siguientes parámetros:

\section{Riqueza conceptual (valor J)}

Hace referencia a la cantidad total de las diferentes palabras definidoras generadas por los grupos bajo estudio, con respecto al término 'psicología médica.'

\section{Conjunto SAM}

Comprende las 20 palabras definidoras con mayor peso semántico para el concepto explorado, que incluye:

- Frecuencia. Se refiere al número de ocasiones en que los alumnos utilizaron una misma palabra al referirse al término 'psicología médica'.

- Valor semántico (valor M). Enumera las palabras definidoras señaladas con mayor frecuencia, en base a la importancia que el estudiante les asigna; por ejemplo, si ocupa el primer lugar en importancia, se le da un valor de 20 puntos, y si ocupa el último lugar, se le atribuye un punto.

- Densidad conceptual (valor G). Se refiere al grado de cercanía o distancia entre las palabras analizadas, calculado en base a la distancia porcentual entre una palabra y otra.

\section{Consenso conceptual (valor Q)}

Se buscan semejanzas conceptuales entre las palabras generadas, con el fin de formar agrupaciones. Se construyen dos dimensiones: una general, con las palabras definidoras que engloban el o los términos a estudiar (por ejemplo, si dentro de la dimensión general se incluye una categoría asociada a valores, entonces se colocarían ahí palabras como 'respeto' y 'ética'), y otra específica, en la que se agrupan las palabras definidoras por su familiaridad entre ellas, es decir, por sus rasgos semejantes o complementarios (por ejemplo, si el término a estudiar es 'libro', en esta dimensión se podría incluir la categoría 'contenido', en donde podrían incluirse palabras como 'dibujos', 'gráficas' y 'letras'). Habrá tantas agrupaciones (categorías) como variedad de palabras definidoras expresadas.

Finalmente, a partir de las palabras definidoras que prevalecieron durante los cinco años de la licenciatura y en base al nivel jerárquico que les asignaron los estudiantes, se estructuró un concepto general para definir la psicología médica.

Se trata de un estudio longitudinal, prospectivo, observacional, con cortes transversales anuales. La primera medición se llevó a cabo al inicio del primer año de la carrera, mientras que las subsecuentes (del segundo al quinto año) se realizaron al final de cada ciclo escolar. La muestra estudiada estuvo formada por un grupo de estudiantes de la carrera de médico cirujano de la Facultad de Medicina de la UNAM, perteneciente al Programa de Alta Exigencia Académica (PAEA). El número de alumnos varió poco de un año a otro, debido al movimiento normal de cada ciclo escolar. De este modo, la muestra estuvo conformada por 62, 58, 61, 44 y 46 estudiantes en el primero, segundo, tercero, cuarto y quinto año, respectivamente.

\section{Resultados}

De 724 palabras obtenidas a lo largo de los cinco años, la riqueza conceptual (valor J) correspondió aproximadamente a un promedio de cinco palabras diferentes por alumno en el primer año, cuatro en el segundo, cinco en el tercero, ocho en el cuarto y seis en el quinto año.

Para el conjunto SAM (valor $M$ en la tabla I), se seleccionaron las 20 palabras definidoras con mayor peso semántico de cada año escolar. De éstas, ocho palabras se mantuvieron a lo largo de los cinco años de la licenciatura: 'mente' (100\%), 'paciente' (66\%), 'relación' (43\%), 'ayuda' (42\%), 'médico' (39\%), 'comprensión' (37\%), 'personalidad' (35\%) y 'salud' (25\%).

$\mathrm{Al}$ inicio del primer ciclo escolar, aparecen las palabras definidoras 'ciencia, 'humanismo', 'estudio', 'conciencia,' 'emociones' y 'psicólogo', que no se presentan nuevamente en el transcurso de la carrera. Ahora bien, después de haber cursado el primer y segundo año, en los que se cursan las asignaturas 
Tabla I. Valores semánticos jerárquicamente asignados por los alumnos cada año a las palabras que utilizaron para definir 'psicología médica'.

\begin{tabular}{|c|c|c|c|c|c|c|c|c|c|c|c|c|c|}
\hline & Palabra & $n$ & $f$ & Valor M & $\%$ & Valor G & & Palabra & $n$ & $f$ & Valor M & $\%$ & Valor G \\
\hline \multirow{20}{*}{ 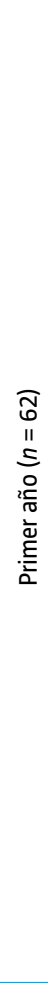 } & Mente & 42 & 68 & 245 & 100 & & \multirow{20}{*}{ 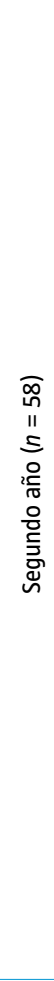 } & Mente & 32 & 55 & 274 & 100 & \\
\hline & Paciente & 26 & 42 & 128 & 52 & 48 & & Paciente & 22 & 38 & 154 & 56 & 44 \\
\hline & Ayuda & 28 & 45 & 123 & 50 & 2 & & Relación & 18 & 31 & 128 & 47 & 9 \\
\hline & Ciencia & 14 & 23 & 122 & 50 & 0 & & Relación M-P & 17 & 29 & 116 & 42 & 4 \\
\hline & Comprensión & 26 & 42 & 100 & 41 & 9 & & Cerebro & 21 & 36 & 104 & 38 & 4 \\
\hline & Humanismo & 13 & 21 & 74 & 30 & 11 & & Médico & 16 & 28 & 86 & 31 & 7 \\
\hline & Salud & 17 & 27 & 69 & 28 & 2 & & Conducta & 13 & 22 & 79 & 29 & 3 \\
\hline & Médico & 14 & 23 & 63 & 26 & 2 & & Personalidad & 21 & 36 & 78 & 28 & 0 \\
\hline & Conducta & 17 & 27 & 57 & 23 & 2 & & Sociedad & 13 & 22 & 57 & 21 & 8 \\
\hline & Personalidad & 17 & 27 & 56 & 23 & 0 & & Comprensión & 9 & 16 & 48 & 18 & 3 \\
\hline & Medicina & 9 & 15 & 46 & 19 & 4 & & Sentimiento & 8 & 14 & 47 & 17 & 0 \\
\hline & Enfermedad & 17 & 27 & 40 & 16 & 2 & & Enfermedad & 13 & 22 & 47 & 17 & 0 \\
\hline & Estudio & 10 & 16 & 38 & 16 & 1 & & Comportamiento & 8 & 14 & 44 & 16 & 1 \\
\hline & Relación & 11 & 18 & 37 & 15 & 0 & & Familia & 13 & 22 & 44 & 16 & 0 \\
\hline & Conciencia & 10 & 16 & 35 & 14 & 1 & & Ayuda & 9 & 16 & 39 & 14 & 2 \\
\hline & Problemas & 9 & 15 & 34 & 14 & 0 & & Carácter & 9 & 16 & 38 & 14 & 0 \\
\hline & Emociones & 12 & 19 & 32 & 13 & 1 & & Salud & 9 & 16 & 37 & 14 & 0 \\
\hline & Sentimiento & 9 & 15 & 25 & 10 & 3 & & Esquizofrenia & 8 & 14 & 15 & 6 & 8 \\
\hline & Psicólogo & 9 & 15 & 22 & 9 & 1 & & Trastorno & 7 & 12 & 12 & 4 & 1 \\
\hline & Terapia & 12 & 19 & 16 & 7 & 2 & & Problemas & 7 & 12 & 7 & 3 & 2 \\
\hline \multirow{20}{*}{ 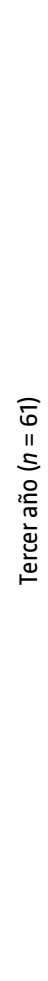 } & Mente & 28 & 46 & 227 & 100 & & \multirow{20}{*}{ 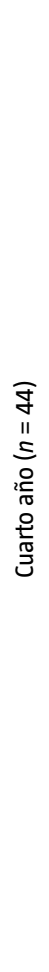 } & Mente & 20 & 45 & 119 & 100 & \\
\hline & Paciente & 31 & 51 & 149 & 66 & 34 & & Paciente & 13 & 30 & 79 & 66 & 34 \\
\hline & Relación & 25 & 41 & 122 & 54 & 12 & & Ayuda & 11 & 25 & 63 & 53 & 13 \\
\hline & Personalidad & 26 & 43 & 120 & 53 & 1 & & Relación M-P & 7 & 16 & 60 & 50 & 3 \\
\hline & Médico & 19 & 31 & 98 & 43 & 10 & & Comprensión & 8 & 18 & 57 & 48 & 3 \\
\hline & Medicina & 10 & 16 & 82 & 36 & 7 & & Relación & 8 & 18 & 49 & 41 & 7 \\
\hline & Cerebro & 17 & 28 & 69 & 30 & 6 & & Transferencia & 7 & 16 & 40 & 34 & 8 \\
\hline & Comprensión & 14 & 23 & 66 & 29 & 1 & & Empatía & 8 & 18 & 38 & 32 & 2 \\
\hline & Salud & 14 & 23 & 63 & 28 & 1 & & Cerebro & 9 & 20 & 36 & 30 & 2 \\
\hline & Ayuda & 12 & 20 & 62 & 27 & 1 & & Pensamiento & 10 & 23 & 35 & 29 & 1 \\
\hline & Depresión & 11 & 18 & 61 & 26 & 1 & & Personalidad & 12 & 27 & 35 & 29 & 0 \\
\hline & Trastorno & 9 & 15 & 61 & 26 & 0 & & Salud & 7 & 16 & 32 & 27 & 3 \\
\hline & Enfermedad & 16 & 26 & 57 & 25 & 1 & & Médico & 8 & 18 & 32 & 27 & 0 \\
\hline & Sociedad & 10 & 16 & 54 & 24 & 1 & & Freud & 6 & 14 & 29 & 24 & 3 \\
\hline & Conocimiento & 11 & 18 & 53 & 23 & 1 & & Psiquiatría & 7 & 16 & 28 & 24 & 1 \\
\hline & Ansiedad & 11 & 18 & 48 & 21 & 2 & & Terapia & 7 & 16 & 28 & 24 & 0 \\
\hline & Tratamiento & 10 & 16 & 41 & 18 & 3 & & Idea & 7 & 16 & 25 & 21 & 3 \\
\hline & Psiquiatría & 10 & 16 & 38 & 17 & 1 & & Depresión & 14 & 32 & 25 & 21 & 0 \\
\hline & Angustia & 11 & 18 & 35 & 15 & 2 & & Duelo & 9 & 20 & 18 & 15 & 6 \\
\hline & Terapia & 10 & 16 & 5 & 2 & 10 & & Ansiedad & 7 & 16 & 5 & 4 & 11 \\
\hline
\end{tabular}


Tabla I. Valores semánticos jerárquicamente asignados por los alumnos cada año a las palabras que utilizaron para definir 'psicología médica' (cont.).

\begin{tabular}{|c|c|c|c|c|c|c|}
\hline & Palabra & $n$ & $f$ & Valor M & $\%$ & Valor G \\
\hline \multirow{20}{*}{ 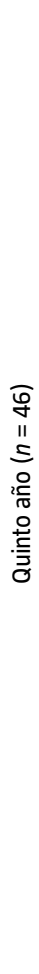 } & Mente & 18 & 39 & 130 & 100 & \\
\hline & Paciente & 11 & 24 & 88 & 68 & 32 \\
\hline & Ayuda & 15 & 33 & 81 & 62 & 5 \\
\hline & Médico & 12 & 26 & 76 & 58 & 4 \\
\hline & Empatía & 11 & 24 & 74 & 57 & 2 \\
\hline & Relación & 10 & 22 & 68 & 52 & 5 \\
\hline & Medicina & 8 & 17 & 60 & 46 & 6 \\
\hline & Comprensión & 14 & 30 & 58 & 45 & 2 \\
\hline & Análisis & 13 & 28 & 55 & 42 & 2 \\
\hline & Relación M-P & 6 & 13 & 52 & 40 & 2 \\
\hline & Personalidad & 14 & 30 & 49 & 38 & 2 \\
\hline & Pensamiento & 8 & 17 & 43 & 33 & 5 \\
\hline & Escuchar & 7 & 15 & 36 & 28 & 5 \\
\hline & Sentimiento & 7 & 15 & 36 & 28 & 0 \\
\hline & Salud & 6 & 13 & 30 & 23 & 5 \\
\hline & Depresión & 11 & 24 & 30 & 23 & 0 \\
\hline & Conducta & 7 & 15 & 24 & 18 & 5 \\
\hline & Cerebro & 7 & 15 & 23 & 18 & 1 \\
\hline & Enfermedad & 7 & 15 & 12 & 9 & 8 \\
\hline & Familia & 7 & 15 & 7 & 5 & 4 \\
\hline
\end{tabular}

$n$ : número de alumnos que asoció la palabra con 'psicología médica'; $f$ : frecuencia con la que se asoció la palabra con psicología médica; valor M: valor semántico jerárquicamente designado; \%: porcentaje del valor $\mathrm{M}$ (el porcentaje del valor M se obtuvo considerando como $100 \%$ el valor M máximo); valor G: grado de cercanía o de dispersión de las palabras (en base a las diferencias entre los porcentajes del valor M; M-P: médico-paciente.

básicas, los estudiantes utilizaron una palabra definidora igual ('problemas') y una semejante ('comportamiento/conducta'); asimismo, sólo en el segundo año se observan las palabras 'comportamiento', 'carácter' y 'esquizofrenia'; 'relación médico-paciente' se observa por primera vez en el segundo ciclo escolar, apareciendo nuevamente en los dos últimos años. En tercer y cuarto año, en los que se cursan las materias en hospitales, los alumnos utilizaron dos palabras definidoras iguales ('ansiedad' y 'psiquiatría'); en tercer año, aparecen por única ocasión las palabras 'conocimiento' y 'angustia', mientras que sólo en el cuarto año se observan las palabras definidoras 'transferencia,' 'Freud, 'idea' y 'duelo'. En los dos últimos años de la licenciatura, los estudiantes utilizaron dos palabras definidoras igua- les ('empatía' y 'pensamiento'). Al finalizar los cinco años de estudio, aparecen nuevas palabras definidoras: 'análisis' y 'escuchar'.

Por último, en el consenso conceptual (valor Q) se formaron dos dimensiones para agrupar las 20 palabras señaladas con mayor frecuencia por los alumnos, con sus respectivas categorías (Tabla II).

\section{Discusión}

Como se esperaba, algunos de los términos utilizados por los alumnos en el primer año para definir 'psicología médica' perduraron a lo largo de los cinco años de la licenciatura. Asimismo, durante ese período, se observaron modificaciones en el orden jerárquico de las palabras (según su valor $M$ ), que no parecen indicar cambios significativos en el modo de pensar de los estudiantes.

En cuanto a las modificaciones que se refieren a la aparición y desaparición de palabras definidoras durante los cinco años de la licenciatura, éstas sí denotan transformaciones en la red conceptual de los alumnos. Estos cambios pueden estar asociados, por un lado, al hecho de permanecer los primeros dos años en una facultad y, posteriormente, asistir a diversos hospitales, y por otra parte, al tipo de asignaturas que se cursan cada año. En este sentido, resulta de interés que a partir del tercer año aparezca por primera vez el término 'depresión' y que permanezca presente durante los siguientes años de la carrera, situación que se explica no sólo por el hecho de que los estudiantes ya han cursado materias asociadas a la psicología médica, sino porque en ese nivel de estudio pueden observar de primera mano ese síntoma en algunos de los pacientes que atienden en la clínica. Destaca también el hecho de que no es hasta el cuarto año de la licenciatura cuando aparece la palabra 'empatía' como definidora del concepto de 'psicología médica'; esto quizá se deba a que la interacción de los alumnos con los pacientes en el contexto hospitalario sensibiliza a los primeros con respecto a la situación y sentimientos que experimentan los segundos. Por otra parte, que palabras definidoras como 'cerebro' aparezcan a partir del segundo año y permanezcan durante el resto de la carrera puede deberse a que, durante los primeros dos años de estudio, los alumnos cursan materias donde comienzan a aprender sobre la anatomía y fisiología de las distintas partes del cuerpo humano, añadiendo a conceptos abstractos como 'mente' (que aparece desde el inicio de la licenciatura y permanece a lo largo de ella) una visión de estructura física, como es el caso del cerebro. 
Tabla II. Agrupación de las palabras utilizadas para definir 'psicología médica' a partir de su semejanza conceptual.

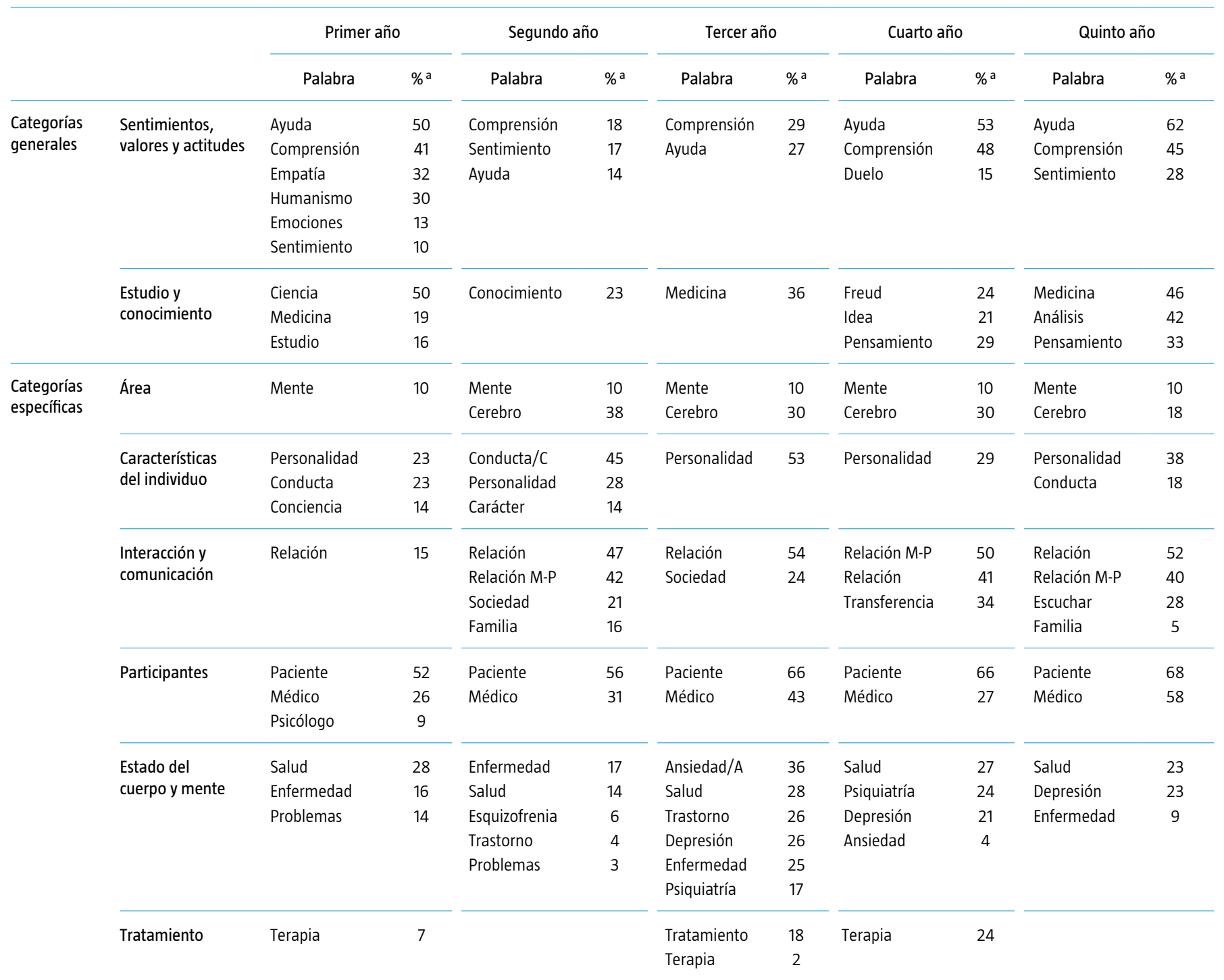

${ }^{\text {a }}$ Porcentaje en base al valor porcentual medio que el alumno dio a cada palabra durante los cinco años (valores M de la tabla I). A: angustia; C: comportamiento; M-P: médico-paciente.

Otro claro indicio de la posible influencia de la enseñanza de una asignatura en la conceptualización que los estudiantes hacen de la psicología médica es la aparición del término 'relación médicopaciente' en el segundo año de la carrera, después de haber cursado la asignatura 'Psicología Médica I', en donde uno de los contenidos curriculares a revisar es precisamente la relación existente entre el médico y su paciente. Esto parece llevar a los alumnos del uso de una palabra más general, como es 'relación' (que está presente desde el inicio hasta el fin de los estudios de los futuros médicos), a reconocer que, dentro de este espectro de la relación, existe un tipo específico que adquiere singular relevancia debido a la forma en que puede incidir en el éxito o fracaso del proceso de la atención médica. En los dos últimos años de la licenciatura reaparece la 'relación médico-paciente' reforzada, al parecer, no sólo después de haber cursado la asignatura 'Psicología Médica II' (donde se retoma el tema sobre la necesidad de que el médico establezca una adecuada relación con su paciente), sino además por la 
convivencia que los alumnos han tenido con los pacientes de los hospitales en donde han continuado sus estudios de medicina.

Se observa que, a lo largo de los cinco años de la carrera, las palabras generadoras 'paciente,' 'médico, 'ayuda, 'relación', 'comprensión' y 'personalidad' no sólo permanecen durante los cinco años de estudio, sino que adquieren mayor relevancia para definir el concepto de 'psicología médica', quizá debido a que asignaturas como 'Psicología Médica I y II', así como 'Psiquiatría', comprenden la enseñanza y aprendizaje de una serie de temas encaminados a formar profesionales de la salud bajo un enfoque humanista, donde el paciente constituye el centro de atención y es visto como individuo integral, debiendo ser el médico sensible a su situación y a los sentimientos que ésta puede generar en él (de ahí que la palabra 'empatía' aparezca en los dos últimos años de la licenciatura como definidora de la psicología médica).

Por otro lado, si bien el término 'salud' se torna de mayor importancia hacia el final de los cinco años de estudio de la medicina, mientras que la palabra definidora 'enfermedad' disminuye su nivel de relevancia semántica para explicar el significado que los estudiantes confieren a la psicología médica, es necesario reforzar en los alumnos una visión más amplia de la medicina y, específicamente, de la psicología médica, donde su práctica profesional no debe orientarse únicamente a remediar la enfermedad, sino que además debe encaminar sus esfuerzos a la promoción de la salud.

Aunque la palabra 'familia' aparece en el segundo año y se presenta nuevamente en el último, los estudiantes no le asignan un valor importante al utilizarla para dar cuenta del significado del concepto de 'psicología médica. Esto puede explicarse porque, a pesar de que tanto en el primer año de la carrera como en el segundo se revisa con los alumnos el tema de la familia como factor esencial a considerar al hablar de la salud o enfermedad del paciente, posiblemente el hecho de que los estudiantes traten con los pacientes en un ambiente hospitalario, donde muchas veces no se involucra a la familia en el proceso de atención del paciente, puede minimizar su perspectiva acerca del papel relevante de la familia como red de apoyo del paciente. Entonces, en el proceso de enseñanza y aprendizaje de los alumnos será necesario considerar estrategias didácticas distintas que no sólo informen, sino que realmente formen y sensibilicen a estos futuros profesionales de la salud en cuanto a la necesidad de involucrar a la familia del paciente en el diagnóstico y atención de éste.
En cuanto al valor $\mathrm{G}$, el hecho de que éste mostrara una mayor distancia porcentual entre las palabras definidoras al iniciar la carrera, da cuenta de que el grupo aún no había desarrollado una cohesión de importancia entre sus miembros con respecto al concepto de 'psicología médica'. A parir del tercer año, al ingresar a los hospitales, continuaba presentándose en el grupo un distanciamiento considerable entre los términos utilizados. Fue sólo en el último año de estudios cuando se observó una cohesión grupal de relevancia entre las palabras generadas por los alumnos. Esta homogeneización del grupo de alumnos en la construcción semántica del término 'psicología médica' permitirá a la institución educativa promover, durante el proceso de formación de los futuros profesionales de la salud, actitudes encaminadas a la aplicación de los principios que sustentan, por ejemplo, la adecuada relación médico-paciente.

Haber llegado a la universidad con un valor $Q$ que denota una visión más panorámica del concepto de 'psicología médica' se debe probablemente al uso abstracto y poco habitual de este término. $\mathrm{Al}$ parecer, con el estudio sistemático de la asignatura 'Psicología Médica', el cúmulo de información que se ha obtenido durante los cinco años sirve para consolidar los términos que permanecen a lo largo de la licenciatura.

En base a las ocho palabras que permanecieron durante todo el estudio, se puede concluir con la siguiente definición jerárquicamente organizada, según la importancia que los estudiantes asignaron a cada palabra generada: 'La psicología médica considera a la mente del paciente dentro de la relación de ayuda por parte del médico, que comprende su personalidad en pro de la salud'. A partir de esta definición, se puede considerar que el grupo de alumnos bajo estudio se licenció con una idea clara de la relevancia que la psicología médica tiene en su práctica profesional.

Por último, cabe aclarar que los resultados de esta investigación se limitan a poblaciones con características similares a las del grupo estudiado.

\section{Bibliografía}

1. López JJ, Ortiz T, López MI. Lecciones de psicología médica. Barcelona: Masson; 1999.

2. Fernández FA. Psicología médica y social. 5 ed. Barcelona: Salvat; 1989.

3. De la Fuente R. Psicología médica. 2 ed. México DF: Fondo de Cultura Económica; 1992.

4. Chur A, Car JE, Bundy C, Sánchez JJ, Tapanya S, Wahass SH. An international perspective on behavioral science education in medical schools. J Clin Psychol Med Settings 2008; 15: 45-53.

5. Soons P, Denollet J. Medical psychology services in Dutch general hospitals: state of the art developments and 
recommendations for the future. J Clin Psychol Med Settings 2009; 16: 161-8.

6. Bordage G. Prototypes and semantic qualifiers: from past to present. Med Educ 2007; 41: 1117-21.

7. Borlawsky T, Friedman C, Lussier YA. Generating executable knowledge for evidence-based medicine using natural language and semantic processing. AMIA Annu Symp Proc 2006; 56-60.

8. Navarro DJ, Griffiths TL, Steyvers M, Lee MD. Modeling individual differences using Dirichlet processes. J Math Psychol 2006; 50: 101-22.

9. Blei DM, Griffiths TL, Jordan MI. The nested Chinese restaurant process and Bayesian nonparametric inference of topic hierarchies. JACM 2010; 57: 1-30.

10. Escobar M. Redes semánticas en textos periodísticos: propuestas técnicas para su representación. Empiria 2009; 17: 13-39.

11. Fitzgerald GA, Doerfel ML. The use of semantic network analysis to manage customer complaints. Commun Res Rep 2004; 21: 231-42.

12. McCray A. An upper-level ontology for the biomedical domain. Comp Funct Genomics 2003; 4: 80-4.

13. Bossomaier T, Harré M, Knittel A, Snyder A. A semantic network approach to the creativity quotient (CQ). Creativity Res J 2009; 21: 64-71.

14. Vera JA, Pimentel CE, Batista FJ. Redes semánticas: aspectos teóricos, técnicos, metodológicos y analíticos. Ra Ximhai 2005; 1: 439-51.

15. Steyvers M, Tenenbaum JB. The large-scale structure of semantic networks: statistical analyses and a model of semantic growth. Cogn Sci 2005; 29: 41-78.
16. Cabalín D, Navarro N. Conceptualización de los estudiantes sobre el buen profesor universitario en las carreras de la salud de la Universidad de La Frontera, Chile. Int J Morphol 2008; 26: 887-92.

17. Collipal E. Conceptualización a través de redes semánticas naturales de los módulos de auto-aprendizaje en anatomía humana. Rev Chil Anat 2002; 20: 63-7.

18. San Martín R. El significado de las categorías 'izquierda' y 'derecha': información, contraste y participación política juvenil. Última Década 2006; 14: 125-49.

19. Trejo H, Camacho R, Herrera J, González A. Significado semántico de lactancia materna y lactancia artificial, en mujeres y hombres. Rev Mex Pediatr 2011; 78: 10-5.

20. Contreras C, López EO. Sobre la representación conceptual y el significado de la autoestima y del concepto de jóvenes. Rev Intercont Psicol Educ 2011; 13: 99-114.

21. Del Navarro RE, Ramírez MSJ. Construyendo el significado del cuidado ambiental: un estudio de caso en educación secundaria. REICE 2006; 4: 52-70.

22. Zermeño AI, Arellano AC, Ramírez VA. Redes semánticas naturales: técnica para representar los significados que los jóvenes tienen sobre televisión, internet y expectativas de vida. ESCC 2005; 11: 305-34.

23. Padilla VM, López EO, Rodríguez MC. Nueva tecnología educativa para evaluar cognitivamente el aprendizaje significativo. CiENCiAUANL 2009; XII: 71-9.

24. González S, Valdez JL. Significado psicológico de la depresión en médicos y psicólogos. Psicología y Salud 2005; 15: 257-62. 